\title{
Thermodynamic modelling of solution and ordered phases
}

\author{
I. Ansara
}

Laboratoire de Thermodynamique et de Physico-Chimie Métallurgiques (UMR 5614) 1130 Rue de la Piscine, 38402 Saint Martin d'Heres Cedex, France

Thermodynamic calculations are successfully used to predict multiphase equilibria or to analyse industrial processes. Different models have been used to evaluate the thermodynamic behaviour of the solution phases, non-stoichiometric compounds with different structures, or phases which present order-disorder transformations. The most common models which are used will be briefly described. The mathematical equivalences between some of them will be emphasized.

\section{Introduction}

In recent years, a great effort was made to represent the thermodynamic properties of solution substitutional phases as well as ordered ones. Many models, empirical or derived from statistical thermodynamics have been published. For solid phases, the models are more and more based on the knowledge of the crystal structure. It is not the aim of this contribution to list all of them but to discuss the mathematical equivalences which can be observed among certain of them, especially for those which have been applied to multcicomponent systems.

\section{$\underline{\text { Substitutional Solutions }}$}

Power series expansions have been used to describe the thermodynamic behaviour of substitutional solutions by Margules (ref. 1). Since then Redlich and Kister (ref. 2), Esdaile (ref. 3), Sharkey (ref. 4) , Bale and Pelton (ref. 5) and Tomiska (ref. 6) used different fomulations listed in Table 1. Conversion matrices of the coefficients have been established (ref. 7,8).

Krupkowski's (ref. 9) empirical equation presents a dissymmetry and it is equivalent to HochArpshofen's equation (ref. 10) which related the interaction parameter to the interatomic bonding strength with respect to the molar fraction of the component with smaller binding capacity.

These power series have been extended to multicomponent systems. However, for such systems, Hillert (ref. 7) suggested that the following composition variable be used:

$$
v_{i j}=\left(1+(1-m) x_{i}-\sum_{j \neq i}^{m} x_{j}\right) / m
$$

Its advantage is that $\sum v_{i j}=1$ whatever the order of the system, and a certain symmetry for the mole fractions is introduced.

Instead of using molar fractions as composition variables, volume fractions were employed like in the equations suggested by Van Laar (ref. 11), Scatchard-Hamer (ref. 12), Flory-Huggins (ref. 13,14), Wohl(ref. 15), and Wilson (ref. 16).

Van Laar, Scatchard-Hamer and Wohl equations are equivalent analytically as seen in Table I. All these equations were used to represent the thermodynamic properties of binary and ternary organic mixtures but very seldom for metallic solutions. This is also the case for the Non-Randon Two Liquid equation (NRTL) developed by Renon and Prausnitz (ref. 17). 
Table 1 - Analytical Equations used for the Representation of the Excess Gibbs Energies of Substitutional Solutions.

\begin{tabular}{|c|c|c|c|}
\hline Equation & Ref. & excess function & Comments \\
\hline Margules & (1) & $\sum_{i=0}^{\nu} a_{i} x^{\nu}$ & \\
\hline Redlich - Kister & $(2)$ & $\sum_{i=0}^{\nu} a_{\nu}\left(x_{i}-x_{j}\right)^{\nu}$ & $x_{i}+x_{j}=1$ \\
\hline Esdaile & (3) & $\begin{array}{l}x_{A} x_{B}\left[\sum_{\nu}\left(A 1_{1(\nu+1)} x_{A}^{\nu-1} x_{B}\right.\right. \\
+\sum_{\nu}\left(A 2_{\left.2(\nu+1) x_{A} x_{B}^{\nu-1}\right]}\right.\end{array}$ & \\
\hline Sharkey et al & (4) & $x_{A} x_{B}\left(\alpha_{1} x_{A}+\alpha_{2} x_{B}-\alpha_{3} x_{A} x_{B}\right)$ & \\
\hline Legendre series & (5) & $\sum_{n=0}^{p} q_{n} P_{n}(x)$ & $\begin{array}{l}P_{n}(\text { Legendre polynomials }) \\
P_{0}=1 \\
P_{1}=2 x-1 \\
P_{n}(x)=[(2 n-1)(2 x-1) / n] P_{n-1}(x) \\
-[(n-1) / n] P_{n-2}(x)\end{array}$ \\
\hline T.A.P. Beries & $(6)$ & $(1-x) \sum_{n=1}^{N} C_{n} x^{n}$ & \\
\hline Krupkowski & (9) & $\alpha\left(x-x^{n}\right)$ & $\begin{array}{l}\text { equivalent to } \\
\text { Hoch - Arpshofen equation (10) }\end{array}$ \\
\hline Van Laar & (11) & $\sum_{i j} a_{i j} z_{i} z_{j}$ & $\begin{array}{l}z_{i}=p_{i} x_{i} / \sum_{j} p_{j} x_{j} \\
p_{i}: \text { constants } \\
S^{x i}=0\end{array}$ \\
\hline Scatchard-Hamer & (12) & $\sum_{i j} a_{i j} z_{i} z_{j}$ & $\begin{array}{l}z_{i}=V_{i} x_{i} / \sum_{j} V_{j} x_{j} \\
S^{x_{s}}=0\end{array}$ \\
\hline Flory - Huggins & $(13,14)$ & $\sum_{i}^{n} x_{i} \ln \left(\phi_{i} / x_{i}\right)$ & $\phi_{i}:$ volumefraction of $\mathrm{i}$ \\
\hline Wohl & $(15)$ & $\sum_{i j} a_{i j} z_{i} z_{j}$ & $\begin{array}{l}q_{i}: \text { effective molar volume of } \mathbf{i} \\
V_{i}: \text { molar volume of } \mathrm{i}\end{array}$ \\
\hline Wilson & $(16)$ & $-\sum_{i}^{n} x_{i} \ln \left[\sum_{j=1}^{n} x_{j} \Lambda_{i j}\right]$ & $H_{m}=0$ \\
\hline
\end{tabular}

A variety of empirical equations based on geometrical weighting to describe the thermodynamic properties of ternary systems have been developed, for example the equations suggested by Kohler (ref. 18), Muggianu (ref. 19), Toop(ref. 20), and Hillert (ref. 7) for multicomponent systems. For a ternary system, the excess Gibbs energy can be expressed in a general form as follows:

$$
G^{x s}=\alpha\left(G_{12}^{x s}\right)_{p}+\beta\left(G_{13}^{x s}\right)_{q}+\gamma\left(G_{23}^{x s}\right)_{r}
$$

where $\alpha, \beta, \gamma$ and $\mathrm{p}, \mathrm{q}, \mathrm{r}$ are given in Table 2 . expression:

Another type of symmetrical equation was suggested by Colinet (ref. 21) which has the following

$$
G^{x s}=\frac{1}{2} \sum_{i=1}^{m} \sum_{j=1}^{m} \frac{x_{i}}{1-x_{j}}\left(G_{i j}^{e x}\right)_{x_{j}}
$$

Kohler, Muggianu and Colinet'S equations are appropriate if the thermodynamic properties of the limiting binary systems are not very different, whereas Toop and Hillert's equations, which are asymmetri- 
Table 2 - Geometrical-type Equations used for the Representation of the Excess Gibbs Energies of Substitutional Solutions.

\begin{tabular}{|l|c|c|c|c|c|c|c|}
\hline Equation & Ref. & $\alpha$ & $p$ & $\beta$ & $q$ & $\gamma$ & $r$ \\
\hline Kohler & $(18)$ & $\left(x_{1}+x_{2}\right)^{2}$ & $x_{1} / x_{2}$ & $\left(x_{1}+x_{3}\right)^{2}$ & $x_{1} / x_{3}$ & $\left(x_{2}+x_{3}\right)^{2}$ & $x_{2} / x_{3}$ \\
Muggianu & $(19)$ & $\frac{x_{1} x_{2}}{v_{12} v_{23}}$ & $x_{1}+\frac{x_{3}}{2}$ & $\frac{x_{1} x_{3}}{v_{12} v_{32}}$ & $x_{1}+\frac{x_{2}}{2}$ & $\frac{x_{2} x_{3}}{v_{21} v_{31}}$ & $x_{2}+\frac{x_{1}}{2}$ \\
Toop & $(20)$ & $\frac{x_{2}}{1-x_{1}}$ & $x_{1}$ & $\frac{x_{3}}{1-x_{1}}$ & $x_{1}$ & $\left(1-x_{1}\right)^{2}$ & $\frac{x_{2}}{x_{3}}$ \\
Hillert & $(21)$ & $\frac{x_{2}}{1-x_{1}}$ & $x_{1}$ & $\frac{x_{3}}{1-x_{1}}$ & $x_{1}$ & $\frac{x_{2} x_{3}}{v_{23} v_{32}}$ & $v_{23}$ \\
\hline
\end{tabular}

$v_{23}=\left(1+x_{2}-x_{3}\right) / 2$

Hillert (ref. 7) has shown that Muggianu's equation (ref. 19) and the Redlich-Kister polynomial (ref. 2) were equivalent. He also showed that assuming the constituent binary systems to be sub-regular, Kohler (ref. 18) and Toop's (ref. 20) equations reduce to a Redlich-Kister type equation but with an extra term.

Very recently, Wang et al. (ref. 22) presented a formalism called the three-factor model generalized later as the multi-factor model for ternary systems(ref. 23), which expresses any excess function, for example the excess Gibbs energy, as follows:

$$
G^{x s}=\sum_{l=1}^{l \prime} \beta_{A B}^{(l)} f_{A B}^{(l)} G_{A B}^{x s}\left(x_{A}^{l}, x_{B}^{l}\right)+\sum_{m=1}^{m^{\prime}} \beta_{A C}^{(m)} f_{A C}^{(m)} G_{A C}^{x s}\left(x_{A}^{m}, x_{C}^{m}\right)+\sum_{n=1}^{n^{\prime}} \beta_{B C}^{(n)} f_{B C}^{(n)} G_{B C}^{x s}\left(x_{B}^{n}, x_{C}^{n}\right)
$$

where $G_{i j}^{x s}$ is the value of the excess Gibbs energy of the binary system $i j$ at the composition $x_{i}$ and $x_{j} . \beta_{i j}^{s}$ is a number $\left(0 \leq \beta_{i j}^{s} \leq 1\right)$ which fulfills the following condition $\sum_{s=1}^{s^{\prime}} \beta_{i j}^{s}=1$.

The geometrical equations described above are compatible with equation 4 . Nevertheless, it has not yet been applied to multicomponent systems.

Specific interaction terms, which are in general of the form $\Pi_{i}^{n} x_{i} P\left(x_{i}\right)$ where $P\left(x_{i}\right)$ is a polynomial, can be added to the excess function in equation 2, the parameters of the polynomial being derived from experimental information.

Power series expansions are derived from statistical thermodynamics if the bonding energies are assumed to be independent of composition or to vary linearly, like for instance in the regular solution model (ref. 24,25 ), the central atom theory (ref. 26), the surrounded atom model (ref. 27) or the surrounded ion model (ref. 28) which was applied to salt mixtures.

Different models were used to describe the thermodynamic properties of the liquid phase, the quasi-chemical model (ref. 29), the associate model (ref. 30), Kapoor-Frohberg (ref. 31) and the ionic liquid model (ref. 32). The latter will be described hereunder. 
The basic assumptions of the quasi-chemical model are that every atom has $z$ nearest neighbours but the competitive action of different types of atom towards a central atom is taken into account. The distribution of the components is calculated using a mass action like expression. As the model takes into account interatomic interactions when determining the most probable configuration of the solution, a chemical ordering effect is thus introduced. This model has been widely used for metallic solutions. It should be noted that the configurational entropy derived from this model is equivalent to the one derived from the cluster variation method developed by Kikuchi (ref. 33,34) where the distribution variable is a basic cluster of lattice points.

As in that model ordering occurs for a composition equal to 0.5 , a modification has been proposed by Pelton and Blander (ref. 35) in order to translate the minima in the entropy or enthalpy functions at any desired compositon. Furthermore, the modified quasi-chemical model has been extended also to multicomponent slag systems.

Many systems exhibit a very negative deviation from ideality for the enthalpy of formation of liquid solutions. A specific model which takes into account the formation of chemical bonding was suggested by Dolezalek (ref. 30). The liquid phase is assumed to contain short range order volume elements, called associates or complexes, which have a well defined composition. They are formed by reaction between the elemental constituents of the phase. Thus, the solution phase will be formed of free atoms and associates, the corresponding number of moles being defined as $n_{A}^{*}, n_{B}^{*}$ and $n_{A_{i} B_{j}}$.

Assuming that the associates are formed by the following reaction

$$
i A+j B \rightleftharpoons A_{\mathrm{i}} B_{\mathrm{j}}
$$

the mole fractions of the various species are defined as

$$
x_{A}^{*}=\frac{n_{A}}{n_{A}+n_{B}+n_{A_{i} B_{j}}}, x_{B}^{*}=\frac{n_{B}}{n_{A}+n_{B}+n_{A_{i} B_{j}}}, x_{A_{i} B_{j}}^{*}=\frac{n_{A_{i} B_{j}}}{n_{A}+n_{B}+n_{A_{i} B_{j}}}
$$

The number of moles of these species can be related to the number of moles of the constituents of a solution where no association occurs, by the following equations:

$$
n_{A}=n_{A}^{*}+i \cdot n^{*} A_{i} B_{j} n_{B}=n_{B}^{*}+i \cdot n^{*} A_{i} B_{j}
$$

By combining equations 5 and 6 , the following relationships are obtained:

$$
\begin{aligned}
& x_{A}^{*}=x_{A}-x_{A_{i} B_{j}}\left[x_{A}(1-i-j)-i\right] \\
& x_{B}^{*}=x_{B}-x_{A_{i} B_{j}}\left[x_{B}(1-i-j)-j\right]
\end{aligned}
$$

The corresponding Gibbs energy per mole of atom, $G_{m}$, is equal to:

$$
\begin{aligned}
G_{m} & =x_{A_{i} B_{j}} G^{a s s}+R T\left(x_{A_{i} B_{j}} \ln x_{A_{i} B_{j}}+x_{A_{i}} \ln x_{A_{i}}+x_{B_{j}} \ln x_{B_{j}}\right) \\
& +x_{A_{i}} x_{B_{j}} \omega_{A_{i}, B_{j}}+x_{A_{i}} x_{A_{i} B_{j}} \omega_{A_{i}, A_{i} B_{j}}+x_{B_{j}} x_{A_{i} B_{j}} \omega_{B_{j}, A_{i} B_{j}}
\end{aligned}
$$

The terms $\omega_{k, l}$ are generally regular solution parameters.

This model has widely been used by several investigators in particular Kehiaian et al. (ref. 36), Jordan(ref. 37), Laugier (ref. 38), Sommer (ref. 39), Yu et al. (ref. 40) with some variants concerning the formulation of the excess Gibbs energy. It had been applied to describe liquid binary or higher order mixtures formed by elements of groups II and VI (ref. 37,38,40), or IV and VI (ref. 38). Sommer (ref. 39) generalized the model to take into account simultaneously different types of associates. 
A statistical thermodynamic treatment was performed by Kapoor and Frohberg (ref. 31) to formulate the activities in silicate melts. The basic assumption is that the mixture is formed of cells which contain one oxygen ion surrounded by cations. The mixing between an oxide $\mathrm{M}_{\mathrm{i}} \mathrm{O}$ and silica $\mathrm{SiO}_{2}$ will then involve symmetrical cells of the type $\mathrm{M}_{\mathrm{i}} \mathrm{OM}_{\mathrm{i}}$, SiOSi and $\mathrm{M}_{\mathrm{i}} \mathrm{OSi}$. Gaye and Welfringer (ref. 41) extended this cellular model to multicomponent systems.

\section{Ordered Phases}

In the solid state, ordered phases having different structures may exist, for example, in metallic systems, the $\sigma-\mathrm{CrFe}$ which is formed of five sub-lattices, or the Laves $\mathrm{C} 14, \mathrm{C} 15$ and $\mathrm{C} 36$ formed respectively by 3,2 and 4 sub-lattices.

A number of phases which can be described with different sub-lattices is listed in Table 3 . Their number and the species occupying them is generally obtained from structural information. The $\mathrm{Ll}_{2}$ and $\mathrm{CsCl}-\mathrm{B} 2$ phases tend to disorder for a given temperature and composition ranges. The substitutional solution is a limiting case where only a single sub-lattice exists.

Table 3 : Different types of phases with sub-lattices

$$
\begin{array}{ll}
\text { Reciprocal Salt System } & \left(A^{+}, B^{+}\right)_{a}\left(C^{-}, D^{-}\right)_{b} \\
\text { III-V Compound } & \left(A^{I I I}, B^{I I I}\right)_{a}\left(C^{V}, D^{V}\right)_{b} \\
\text { Interstitial Solution } & (F e, N i)_{a}(C, N, \square)_{b} \\
\text { Ordered Phase i.e. } L 1_{2} & (A l, N i)_{3}(A l, N i) \\
\text { Ordered Phase i.e. } B_{2} & (A l, N i)(\square, N i) \\
\text { Ionic Liquid Solution } & \left(A l^{+3}\right)_{p}\left(O^{-2}, S i O_{4}^{-4}, S i O_{2}^{0}\right)_{q} \\
\text { Substitutional Solution } & \left(A_{x_{1}} B_{x_{2}} C_{x_{3}} \ldots \ldots \ldots . . . .\right)
\end{array}
$$

These phases can be schematically described as follows:

$$
\left(A_{y_{A}^{\prime}} B_{y_{B}^{\prime}} \ldots \ldots\right)_{p}\left(A_{y_{A}^{\prime \prime}} B_{y_{B}^{\prime \prime} \cdots \cdots}\right)_{q} \cdots
$$

where the species $A^{\prime}, B^{\prime} \ldots$ can be atoms, vacancies, defects, ions.... $p, q \ldots$ are the number of sites. If $p+q+\ldots=1$, then the thermodynamic quantities are referred to one mole of sites.

For each sub-lattice $s$, the site fraction of the species $i$ is equal to

$$
y_{i}^{s}=\frac{n_{i}^{s}}{\sum_{j} n_{j}^{s}}=\frac{n_{i}^{s}}{n^{s}}
$$

$$
\text { with } \quad \sum_{k} y_{k}^{s}=1 \quad \text { and } \quad \sum_{k} n^{s}=n \text {. }
$$

$n_{j}^{s}$ is the number of species $j$ in sub-lattice $s, n^{s}$ the number of sites in sub-lattice $s$, and $n$ the total number of sites. $n^{s}$ is related to $n$ by $n^{s}=p \cdot n /(p+q+\ldots)$.

The sub-lattice model, developed by Hillert and Staffansson (ref. 42) based on Temkin's model for ionic solutions (ref. 43) and extended by Sundman and Agren (ref. 44) is very convenient to decribe the thermodynamic properties of the ordered phases. The molar Gibbs energy is expressed by the following equation:

$$
G_{m}=G-\sum_{i} x_{i} G_{i}^{\varphi}=G^{r e f}+G^{i d}+G^{x s}
$$

where $G_{m}$ is referred to the pure elements in a given physical state at a given temperature and pressure. 
Gibbs energy of configuration, $G_{m}^{\text {id }}$

The term $G_{m}^{\text {id }}$ is related to the molar entropy of configuration $S_{m}^{\text {conf }}$. For the general case of a multicomponent system formed by several sub-lattices, each of them containing $j$ species interchanging randomly, the number of permutations is given by the following equation:

$$
W_{p}=\left[\prod_{s} \frac{n^{s !}}{\prod_{j} n_{j}^{s !}}\right]
$$

$S_{m}^{\text {conf }}$ can then be derived and is equal to

$$
S_{m}^{c o n f}=\frac{S^{c o n f}}{N_{a} \cdot n}=-R \sum_{i} u^{*} \sum_{i} y_{i}^{a} \ln y_{i}^{s}
$$

This equation also applies to a substitutional or associate solutions. The expressions of the configurational entropy can become more and more complicated, as for example in the case where species are replaced by clusters, for example in the Cluster Variation Method (ref. 35). In the case of a four-point tetrahedron considered as a basic cluster, for a system of $n$ lattice points, the number of permutations between points, pairs and tetrahedrons is equal to:

$$
W_{p}=k n \frac{\left[\prod_{i, j}\left(y_{i j}\right) !\right]^{6} n !}{\left[\prod_{i, j, k, l}\left(z_{i j k l} N\right) !\right]^{2}\left[\prod_{i}\left(x_{i} n\right) !\right]^{5}}
$$

\section{Surface of Reference}

The term $G^{\text {ref }}$ defines a surface of reference. For a two sub-lattice phase of the type $\left(A_{y_{A}^{\prime}} B_{y_{B}^{\prime}}\right)_{p}\left(C_{y_{C}^{\prime}} B_{y_{D}^{\prime}}\right)_{q}$ taken as an example, $G^{\text {ref }}$ is equal to

$$
G^{r e f}=y_{A}^{\prime} y_{C}^{\prime \prime} G_{A_{p} C_{q}}+y_{A}^{\prime} y_{D}^{\prime \prime} G_{A_{p} D_{q}}+y_{B}^{\prime} y_{C}^{\prime \circ} G_{B_{p} C_{q}}+y_{B}^{\prime} y_{D}^{\prime \prime} G_{B_{p} D_{q}}
$$

The terms $G_{i_{p} j_{q}}$ represent the Gibbs energy of formation of the "ideal compound". It should be noted that in the case of a substitutional solution equation 9 is equal to zero. Some of these compounds may be metastable.

\section{Excess Gibbs Energy $G_{m}^{x s}$}

The excess Gibbs energy $G_{m}^{x s}$ is equal to

$$
\begin{aligned}
G_{m}^{x s}= & y_{A}^{\prime} y_{B}^{\prime}\left[y_{A}^{\prime \prime} L_{A, B: C}+y_{B}^{\prime \prime} L_{A, B: D}\right]+y_{A}^{\prime \prime} y_{B}^{\prime \prime}\left[y_{A}^{\prime} L_{A: C, D}+y_{B}^{\prime} L_{B: C, D}\right] \\
& +y_{A}^{\prime} y_{B}^{\prime} y_{A}^{\prime \prime} y_{B}^{\prime \prime} L_{A, B: C, D}
\end{aligned}
$$

The terms $L_{i, j: k}$ and $L_{i: k, l}$ represent the interaction parameters between the atoms on one sub-lattice for a given occupancy of the other, and they can be described by a Redlich-Kister (ref. 2) type polynomial, as follows:

$$
L_{i, j: k}=y_{i} y_{j}\left[\left(a_{0}+b_{0} T+\left(a_{1}+b_{1} T\right)\left(y_{i}-y_{j}\right)\right]\right.
$$

For ordered compounds exhibiting narrow ranges of non-stoichiometry, like for example the Cscl-B2 phase which exists in the Al-Ni system, an atom B replaces an atom A on the first sub-lattice, (called antistructure by Wagner (ref. 45)), and a defect, in this case a vacancy, occupies the site of a B atom in the second sub-lattice. A schematic way of illustrating that phase is the following:

$$
\left(A_{y_{A}^{\prime}} B_{y_{B}^{\prime}}\right)_{p}\left(\square_{y_{a}^{\prime \prime}} B_{y_{B}^{\prime \prime}}\right)_{q} \quad\left(A_{y_{A}^{\prime}} B_{y_{B}^{\prime}}\right)_{p}\left(\square_{y_{A}^{\prime \prime}} B_{y_{B}^{\prime \prime}}\right)_{q}
$$


In the case of the CsCl-B2 phase, Wagner (ref. 45) expressed the molar Gibbs energy of the phase as

$$
G_{m}=G^{i d}+G_{f}
$$

If the number of defects is small compared to the total number of sites, then $G_{f}$ (enthalpy or total energy as defined by Wagner) is expressed as a linear function of the number of defects in the different sites, as follows:

$$
G_{f}=G^{*}+\frac{n_{B}^{\prime}}{n} G_{2 \bullet}+\frac{n_{\square}^{\prime \prime}}{n} G_{2 \square}
$$

where $G^{*}$ is the Gibbs energy of formation of the ideal compound, $G_{2 \bullet}$ and $G_{2 \square}$ are respectively the Gibbs energy of formation of an anti-structure and of a vacancy.

Ansara et al. (ref. 46) showed that equation 17 is mathematically equivalent to equation 13 and that the identification of terms shows that

$$
\begin{aligned}
& G_{A: B}=G^{*} \\
& G_{A: \square}=G^{*}+q \cdot G_{2 \square} \\
& G_{B: B}=G^{*}+p \cdot G_{2 \bullet} \\
& G_{B: \square}=G^{*}+p \cdot G_{2 \bullet}+q \cdot G_{2 \square}=-G_{A: B}+G_{A: \square}+G_{B: B}
\end{aligned}
$$

The interesting feature resulting from this identification is that the enthalpies of formation of defects as defined by Wagner (ref. 45), that is substitution of an atom by a vacancy on one sub-lattice and by a foreign atom on the other one, are related to the lattice stabilities of hypothetical compounds in the sublattice model. At present, there is a great lack of information for this type of parameter and evaluations are desirable.

Henig et al. (ref. 47) introduced extra terms to equation 17 to account for deviations from linearity. Krachler et al. (ref. 48) generalized the model by considering substitutional antistructure atoms and vacancies on both sub-lattices. The sites are then equivalent and the number of Gibbs energy of formation parameters increases from four to six. The two extra ones are $G_{A: A} G_{\square: 0}$ which are respectively the Gibbs energy of element $A$ in the structure bcc-A2 with respect to a bcc-A2 reference state, and that of the vacancies which can be set to zero. This description is also valid for other types of ordered structures which present a range of non-stiochiometry.

The two sub-lattice model has been applied to more complicated solid phases. For the spinel phases in the $\mathrm{MgO}-\mathrm{Al}_{2} \mathrm{O}_{3}$ system where the cations occupy tetrahedral and octahedral sites, Hallstedt (ref. 49) modelled the phase as $\left(\mathrm{Al}^{3+}, \mathrm{Mg}^{2+}\right)\left(\mathrm{Al}^{3+}, \mathrm{Mg}^{2+}\right)_{2}\left(\mathrm{O}^{2-}\right)_{4}$. For semi-conductor compounds like GaAs, Chen et al. (ref. 50) modelled the phase including lattice defects, free electrons and holes as $\left(A, B, B^{+}\right)\left(B, A, A^{-}\right)(\square, e)(\square, h)$. They also showed that the model is equivalent to the species chemical potential-bond energy model described by Oates $e t$ al. (ref. 51).

Hillert et al. (ref. 32) applied the two sub-lattice model for molten solutions which present different tendencies of ionisation. The configurational entropy is based on Temkin's expression. To account for off-stoichiometric compositions, Hillert $e t$ al. introduce in the anion sub-lattice vacancies and neutral species, for example $(\mathrm{A})_{P}(\mathrm{~B}, \mathrm{~b}, \square)_{Q}$ where $\mathrm{A}, \mathrm{B}, \mathrm{b}$ and $\square$ are respectively cations, anions, neutral species and vacancies. The Gibbs energy per mole of atoms, is expressed as follows:

$$
\begin{aligned}
G_{m}= & {\left[y_{B} \Delta^{\circ} G_{A-\nu_{B} j_{\nu_{A}} B}\right.} \\
& +\mathrm{R} T\left(y_{B} \ln y_{B}+y_{b} \ln y_{b}+y_{\square} \ln y_{\square}\right)+ \\
& \left.+y_{B} y_{b} L_{B, b} / \nu_{A}+y_{B} y_{\square} L_{B, \square} / \nu_{A}+y_{b} y_{\square} L_{b, \square}\right]\left(1-y_{B} \nu_{B} / \nu_{A}\right)
\end{aligned}
$$


$\nu_{i}$ and $\nu_{j}$ are the valency of ions $i$ and $j$. The terms $P$ and $Q$ which are the number of sites on the cation and anion sub-lattices, vary with composition in order to always maintain electroneutrality. They are respectively equal to $\sum_{j}\left(-\nu_{j} y_{j}\right)+y_{\square} Q$ and $\sum_{i}\left(\nu_{i} y_{i}\right) . \Delta^{\circ} G_{A_{-\nu_{B}} / \nu_{A} B}$ is the Gibbs energy of formation of the compound. if $j=1$ with

Hillert et al. (ref. 52) have also shown that this equation is mathematically equivalent to equation 7

$$
\begin{array}{llll}
x_{A_{i} B_{1}} & =y_{B} & x_{A_{1}} & =y_{\square} \\
\nu_{A} G^{a s s} & =\Delta^{\circ} G_{A-\nu_{B} B_{\nu_{A}}} & \omega_{A_{1}, B_{1}} & =L_{b, \square} \\
\nu_{A} \omega_{A_{1}, A_{i} B_{1}} & =L_{B, \square} & \nu_{B} \omega_{B_{1}, A_{i} B_{1}} & =L_{B, b}
\end{array}
$$

Liquid silicates were modelled, with this description. For example in the $\mathrm{Ca}-\mathrm{Fe}$-O-Si system, Selleby (ref. 53) used $\left(\mathrm{Ca}^{2+}, \mathrm{Fe}^{2+}, \mathrm{Si}^{4+}\right)_{P}\left(\mathrm{O}^{2}-, \mathrm{SiO}_{4}^{O 4-}, \square, \mathrm{FeO}_{1.5}, \mathrm{SiO}^{2}\right)_{Q}$.

\section{Modelling of order-disorder transformations.}

The ordered $\mathrm{L}_{2}$ or $\mathrm{DO}_{19}$ structures based on an fcc-A1 or hcp-A3 lattice can disorder at a given composition or temperature. The Gibbs energy of formation of these phases is expressed as follows

$$
G_{m}=G_{m}^{o r d}\left(y_{i}^{\prime}, y_{i}^{\prime \prime}\right)+G_{m}^{d i s}\left(x_{i}\right)
$$

Several models have been used to describe their thermodynamic properties, for example the $\mathrm{Ll}_{2}$ phase in the Al-Ni system. Kaufman et al. (ref. 54) used a substitutional model, hence considering only one sub-lattice. Jia (ref. 55) described the $\mathrm{Ll}_{2}$ as two solutions $(\mathrm{B})_{3}\left(\mathrm{~A}_{y_{A}^{\prime \prime}}, \mathrm{B}_{y_{B}^{\prime \prime}}\right)$ for $x_{A}<0.5$ and $\left(\mathrm{A}_{y_{A}^{\prime}}, \mathrm{B}_{y_{B}^{\prime}}\right)_{3}(\mathrm{~A})$ for $x_{A}>0.5$. The disordered phase was considered to be a separate phase. Ansara et al. (ref. 56) and more recently, Dupin et al. (ref. 57) described the $\mathrm{L}_{2}$ phase as containing both elements in both sublattices $\left(A_{y_{A}^{\prime}}, B_{y_{B}^{\prime}}\right)_{3}\left(A_{y_{A}^{\prime \prime}}, B_{y_{B}^{\prime \prime}}\right)$ but mathematical constraints were establised in order to describe with the same equation the disordered state. The cluster variation method, developed by Kikuchi (ref. 33,34), was used by Sanchez et al. (ref. 58), Sigli et al. (ref. 59), Carlson et al. (ref. 60), Pasturel et al. (ref. 61), and Sluiter et al. (ref. 62), to describe the $\mathrm{NiAl}$ and $\mathrm{Ni}_{3} \mathrm{Al}$ phases, in a tetrahedron or a tetrahedron - octahedron approximation. Lennard - Jones, coherent potential approximation or first-principles calculations were used to estimate the energy parameters.

In equation 19 , the term $G_{m}^{\text {ord }}\left(y_{i}^{\prime}, y_{i}^{\prime \prime}\right)$ can be expressed by equation 13 . The molar fractions of the various elements $x_{i}$ are related to the various site fractions of the same elements $x_{i}=\frac{3}{4} y_{i}^{\prime}+\frac{1}{4} y_{i}^{\prime \prime}$. The phase disorders when $x_{i}=y_{i}^{\prime}=y_{i}^{\prime \prime}$. To ensure that the disordered state is always possible, constraints have to be imposed on the coefficients, and it is sufficient to require that the Gibbs energy should always have an extremum, that is $d G=0$ when $x_{i}=y_{i}^{\prime}=y_{i}^{\prime \prime}$, constraints between the parameters are then obtained (ref. 57) which have to be fulfilled.

For practical use, it is interesting to consider the disordered state independently from the ordered one for a given phase. This is done by splitting the ordering energy into two terms as follows:

$$
G_{m}^{\text {ord }}\left(y_{i}^{\prime}, y_{i}^{\prime \prime}\right)=G_{m}^{o r d, *}\left(y_{i}^{\prime}, y_{i}^{\prime \prime}\right)-G_{m}^{\text {ord,*}}\left(y_{i}^{\prime}=x_{i}, y_{i}^{\prime \prime}=x_{i}\right)
$$

where $G_{m}^{\text {ord,*}}\left(y_{i}^{\prime}, y_{i}^{\prime \prime}\right)$ the Gibbs energy is described by the model 19 and which contains implicitly a contribution to the disordered state, and $G_{m}^{o r d}, *\left(y_{i}^{\prime}=x_{i}, y_{i}^{\prime \prime}=x_{i}\right)$, a term which represents the energy contribution of the disordered state to the ordered phase. When the site fractions are equal in both sub-lattices, thus corresponding to a disordered phase, then equation 20 is equal to zero. Hence, the parameters of both ordered and disordered phases can be evaluated independently. This equation has been implemented in the Thermo-Calc package developed by Sundman et al. (ref. 63). 
The two sub-lattice model is not adapted to describe several order-disorder phases based on the same lattice which can co-exist as in the Au-Cu system, where two $\mathrm{Ll}_{2}$ and $\mathrm{Ll}_{0}$ phases are in equilibrium.

A four sub-lattice model, suggested by Sundman (ref. 64) allows representation of these phases as well as the disordered fcc-Al phases to be described with the same model

$$
\left(A_{y_{A}^{(1)}} B_{y_{B}^{(1)}}\right)\left(A_{y_{A}^{(2)}} B_{y_{B}^{(2)}}\right)\left(A_{y_{A}^{(3)}} B_{y_{B}^{(3)}}\right)\left(A_{y_{A}^{(4)}} B_{y_{B}^{(4)}}\right)
$$

where the four sites are equivalent. The disordered state is described when the site fractions of the different species are the same in the four sub-lattices. If two sub-lattices have the same site fractions, as well as the two others but of diferent values, the model describes then the $\mathrm{L} 1_{0}$ ordering. If three sub-lattices have the same site fractions, different from the fourth, then the $\mathrm{Ll}_{2}$ ordering can be described. This second case will be discussed here.

The Gibbs energy of the ordered phase is expressed by the following equation:

$$
G_{m}=\sum_{i=A}^{B} \sum_{j=A}^{B} \sum_{k=A}^{B} \sum_{l=A}^{B} y_{i}^{(1)} y_{j}^{(2)} y_{k}^{(3)} y_{i}^{(4)} G_{i: j: k: l}+\frac{R T}{4} \sum_{s=1}^{4} y_{i}^{s} \ln y_{i}^{s}+\sum_{s=1}^{4} y_{A}^{s} y_{B}^{s}\left[{ }^{0} L+{ }^{1} L\left(y_{A}^{s}-y_{B}^{s}\right)\right]
$$

The terms $G_{i: j: k: l}$ relative to the same stoichiometry are identical whatever the occupation of the sub-lattice. In addition, the interactions between species in a given sub-lattice are assumed to be independent of the site occupations in the others, and identical in whatever sub-lattice the interaction takes place. Noting that $y_{i}^{(1)}=y_{i}^{(2)}=y_{i}^{(3)}=y_{i}^{\prime}$ and that $y_{i}^{(4)}=y_{i}^{\prime \prime}$, Dupin et al. (ref. 57) have shown that there is a mathematical equivalence between the Gibbs energy derived from the four sub-lattice model with equation 13 for the two sub-lattices. The relationships between the parameters are the following:

$$
\begin{aligned}
& G_{A: B}=G_{A: A: A: B} \quad G_{B: A}=G_{B: B: B: A} \\
& { }^{0} L_{A: A, B}={ }^{0} L \quad{ }^{1} L_{A: A, B}={ }^{1} L \\
& { }^{0} L_{B: A, B}={ }^{0} L \quad{ }^{1} L_{B: A, B}={ }^{1} L \\
& { }^{0} L_{A, B: A}=-1.5 G_{A: B: B: B}+1.5 G_{A: A: B: B}+1.5 G_{A: A: A: B}+3.0^{\circ} L \\
& { }^{0} L_{A, B: B}=1.5 G_{A: B: B: B}+1.5 G_{A: A: B: B}-1.5 G_{A: A: A: B}+3.0^{\circ} \mathrm{L} \\
& { }^{1} L_{A, B: A}=0.5 G_{A: B: B: B}-1.5 G_{A: A: B: B}+1.5 G_{A: A: A: B}+3.0^{1} L \\
& { }^{1} L_{A, B: B}=-1.5 G_{A: B: B: B}+1.5 G_{A: A: B: B}-0.5 G_{A: A: A: B}+3.0^{1} L \\
& { }^{0} L_{A, B: A, B}=0.0
\end{aligned}
$$

The number of variables can be reduced if $G_{A: A: A: B}=G_{B: B: B: A}$ is assumed.

Evaluation of the paramaters

By means of optimisation procedures, the coupling of the experimental thermodynamic information with phase diagram data can lead to optimal values of the thermodynamic properties of the various phases in the system. These procedures use as input data all available experimental information on phase diagram and thermodynamic quantities like activities, enthalpies of formation and heat capacities. They are integrated in several software packages like BINGSS and TERGSS (ref. 65,66), PARROT (ref. 67), $F^{*} A^{*} C^{*} T$ (ref. 68) or CHEMSAGE (ref. 69) which are used worldwide and they provide an excellent representation of the thermodynamic properties of the various phases of a system and are they consistent with phase diagram information. 


\section{Conclusions}

The models described in this contribution have been used with success to describe different types of solution or ordered phases. Many of them are used in calculation programs for multicomponent phase equilibria, as well as in simulation processes. However there is still a lack of exprimental information on multicomponent systems and there is a need on thermodynamic properties for metastable phases.

\section{References}

1. M. Margules, Sitzb. d. mathem.-naturw. C1.,CIV.Bd., Abth., II.a., 104, 1243 (1895).

2. O. Redlich and A. Kister Ind. Eng. Chem. 40, 345 (1948).

3. J. D. Edsaile. Metall. Trans. 2, 2277 (1971).

4. R. L. Sharkey. M. J. Pool and M. Hoch, Metall. Trans. 23039 (1971).

5. C. W. Bale and A.D. Pelton. Metall. Trans. 5, 2323 (1974).

6. J. Tomiska. Calphad. 10, 1,91 (1986).

7. M. Hillert. Calphad. 10, 1, 1 (1980).

8. J. Tomiska. Calphad. 10, 1,91 (1980).

9. A. Krupkowski. Bull. Acad. Pol. Sc. Lett. 1, 15 (1951).

10. M. Hoch and I. Arpshofen. Z. Metallkde. 75, 23 (1984).

11. J. J. Van Laar. Z. Phys. Chem. 83215 (1913).

12. G. Scatchard and W.J. Hamer. J. Amer. Chem. Soc. 71805 (1935).

13. P. J. Flory. J. Chem. Phys. 1051 (1942).

14. M. L. Huggins. Ann. N.Y. Acad. Sci. 431 (1942).

15. K. Wohl. Trans. Am. Inst. Chem. Eng. 42215 (1946).

16. G. M. Wilson. J. Amer. Chem. Soc. 86127 (1964).

17. H. Renon and J. M. Prausnitz. AJChE J. 14, 1, 135 (1968).

18. F. Kohler. Monatsh. Chem. 91,738 (1960).

19. Y. M. Muggianu, M. Gambino and J.-P. Bros. J. Chim. Phys. 22, 83 (1975).

20. G. W. Toop. Trans. AIME. 233, 850 (1985).

21. C. Colinet. D.E.S. Univ. Grenoble. France (1967).

22. Z. C. Wang, R. Lück and B. Predel. Calphad. 14, 3, 217 (1990).

23. Z. C. Wang, R. Lück and B. Predel. Calphad. 14, 3, 235 (1990).

24. J. H. Hildebrand. Proc. Natl. Acad. Sci. Washington, 13, 167 (1927).

25. J. H. Hildebrand. J. Amer. Chem. Soc. 51, 66 (1929).

26. C. H. P. Lupis. Ph.D. Thesis. M.I.T. Boston, U.S.A. (1965).

27. J. C. Mathieu, F. Durand and E. Bonnier. J. Chim. Phys. 11-12, 1289 (1965) and 11-12, 1297 (1965).

28. M. Gaune-Escard, J. C. Mathieu, P. Desre and Y. Doucet. J. Chim. Phys. 9, 1390 (1972) and 9, 1397 (1972).

29. E. A. Guggenheim., Mixtures, Clarendon Press, Oxford, U.K. (1952).

30. F. Dolezalek., Z. Phys. Chem. 64, 727 (1908).

31. M. L. Kapoor and M. G. Frohberg., Chemical Metallurgy of Iron and Steel p. 17, The Iron and Steel Institute, London, U.K. (1973).

32. M. Hillert, B. Jansson, B. Sundman, and J. Ågren. Metall. Trans. 16A, 261 (1985).

33. R. Kikuchi. Phys. Rev. 81, 6, 988 (1951).

34. R. Kikuchi and D. de Fontaine. Proc. Applications of Phase Diagrams in Metallurgy and Ceramics. C. Carter Editor, p.967 NBS Special Pb. 496, Washington (1978).

35. A. D. Pelton and M. Blander. Metall. Trans. 17B 805 (1986).

36. H. V. Kehiaian and W. Treszczanowicz. Bull. Soc. Chim. Fr. 5, 1561 (1969).

37. A. S. Jordan. Metall. Trans. 1, 239 (1970).

38. A. Laugier. Rev. Phys. Appl. 8, 259 (1973). 
39. F. Sommer. Z. Metallkde. 73, 2, 72 (1982).

40. T. C. Yu. and R.F. Brebrick, J. Phase Equil. 13, 5, 476 (1992).

41. H. Gaye and J. Welfringer. Metallurgical Slags and Fluxes, H.A. Fine and D.R. Gaskell, Editors, p. 357, The Metallurgical Society of AIME, Warrendale, Pa. (1984).

42. M. Hillert and L.-I. Staffanson. Acta Chem. Scand. 24, 3618 (1970).

43. M. Temkin. Acta Phys. Chim. 20, 411 (1970).

44. B. Sundman and J. Ágren. J. Phys. Chem. Solids 42, 297 (1981).

45. C. Wagner, Thermodynamics of Alloys. p.54, Addison-Wesley Press Inc., Cambridge, Mass. USA (1952)

46. I. Ansara, N. Dupin, H.-L. Lukas and B. Sundman. Applications of Thermodynamics in the Synthesis and Processing of Materials, P. Nash and B. Sundman Editors, p.273, TMS Warrendale (1995).

47. E. - Th. Henig, H.L. Lukas and G. Petzow. Z. Metallkde. 73, 2, 87 (1982).

48. R. Krachler, H. Ipser, and K.L. Komarek. J. Phys. Chem. Solids. 50, 1127 (1989).

49. B. Hallstedt, J. Am. Ceram. Soc. 75, 6, 1497 (1992).

50. Q. Chen and M. Hillert. J. Alloys and Compounds. 245, 125 (1996).

51. W. A. Oates, G. Eriksson and H. Wenzl. J. Alloys and Compounds. 220, 48 (1995).

52. M. Hillert and J. Ágren. Z. Metallkde. 77, 12, 796 (1986).

53. M. Selleby. TRITA-MAC-0510, Materials Research Center, Royal Institute of Technology, Sweden (1993).

54. L. Kaufman and H. Nesor. Calphad. 2, 4, 325 (1978).

55. C.C. Jia. Doctoral Thesis, Tohoku Univ., Sendai, Japan (1990).

56. I. Ansara, B. Sundman, and P. Willemin. Acta Metall. 36, 4, 977 (1988).

57. N. Dupin, I. Ansara, H. -L. Lukas and B. Sundman. J. Alloys and Compounds. 247, 1-2, 20 (1997).

58. J. M. Sanchez, J.R. Barefoot, R.N. Jarrett, and J.K. Tien. Acta Metall. 32, 9, 1519 (1984).

59. C. Sigli, and J.M. Sanchez. Acta Metall. 33, 6, 1097 (1985).

60. A. E. Carlsson, and J.M. Sanchez. Solid State Comm. 65, 6, 527 (1988).

61. A. Pasturel, C. Colinet, and A.T. Paxton. J. Phys. Condens. Matter. 4, 4, 945 (1992).

62. M. Sluiter, P.E.A. Turchi, F.J. Pinski, and G.M. Stocks. J. Phase Equil. 13, 6, 605 (1992).

63. B. Sundman, B. Jansson and J.-O. Andersson. Calphad. 2, 9, 153 (1985).

64. B. Sundman, private communication

65. H.L. Lukas, B. Zimmerman and E.-Th. Henig. Calphad. 13, 225 (1977).

66. H.L.Lukas and S. Fries. J. Phase Equil. 13. 5, 532 (1992).

67. B. Jansson. Ph.D. Thesis. KTH, Stockholm, Sweden (1983).

68. C.W. Bale, A.D. Pelton and W.T. Thompson. $F^{*} A^{*} C^{*} T$, User's Guide Suppl. (1981), Mc Gill Univ./Ecole Polytechnique, Montreal, Canada.

69. E. Königsberger and G. Eriksson. Calphad 19, 2, 207 (1995). 